\title{
UNSTEADY PERTURBED FLOW AT LOW MACH NUMBER OF A VISCOUS COMPRESSIBLE FLUID
}

\author{
BY \\ CARLO FERRARI \\ Aeronautica Lab, Politecnico, Torino, Italy
}

\begin{abstract}
Summary. The problem of the unsteady perturbed two-dimensional flow at low Mach number of a viscous compressible fluid is studied taking the relation between the stress and deformation rates tensors that was obtained and applied in [1] and [3]. It is shown that the system of equations describing the phenomenon is totally hyperbolic and therefore the perturbations in any point $P$ of the field are propagated by means of waves corresponding to four characteristic surfaces passing through $P$. The displacement and propagation velocities of these waves are determined as well as their dependence on the orientation of their front in $P$; it is shown besides that the discontinuity vector across the waves has components both longitudinal and transversal. The variation laws of the fluid velocities both on the characteristic surfaces and along their bicharacteristics are determined, which allows us to solve the Cauchy problem with a "step-by-step" method.
\end{abstract}

1. Introduction. In (1) I have proved that the paradox of the instantaneous propagation of any small perturbation in a viscous compressible fluid is removed if one takes the relation between the stress and deformation rates tensors, not that given by the classical theory, but that derived by taking into account the dependence on the time of the distribution function of the molecular velocities, in a way similar to that applied by C. Cattaneo in the analogous problem of heat transfer [2]. In [3] I have studied the same problem considered in [1], taking into account the effect of the thermal conductivity on the phenomenon and therefore not assuming this as isentropic. Also in this case, the system of equations defining the problem is totally hyperbolic; it is rather more complicated and, as a consequence of the greater number of equations, the number of the propagation waves is also greater, four instead of two, and they are, two by two, characterized by different displacement velocities. In both these papers the fluid flow, in which the perturbation is generated, is assumed to be uniform and one-dimensional; it has seemed therefore to be of some interest to study how the phenomenon of propagation of small perturbations varies if the fluid flow is a plane flow, being the components of the velocity functions of both space coordinates and of time; it is assumed that the Mach number $M_{0}$ of the flow is small enough in order that the terms of order of magnitude $M_{0}^{2}$ can be ignored. Finally, as in [1], the thermal transfer is not taken into account. 
The results that are obtained seem to be interesting enough: the system of equations is totally hyperbolic; in each point $P$ of the field pass four characteristic surfaces, that are the envelope of the corresponding Monge cones, and that represent the propagation waves, which have different displacement speeds, that are very near to those obtained in [2] at least for a Prandtl number one. These waves, however, are not only longitudinal, since the discontinuity vector across their front has components both longitudinal and transversal. The variation laws of the velocity both on the characteristic surfaces and along their bicharacteristics are determined, which allows us to solve the Cauchy problem with a "step-by-step" method in an analogous but much more complicated way than that indicated in [1] and [3]. On the other hand, the increase in the number of waves having propagation speed variable with the space and time coordinates and affecting each other in the course of their propagation must easily cause a flow field that is very complicated or quite chaotic as a consequence of the generation of discontinuity surfaces for the rotation vector of the field and for the derivatives of deformation rates, which can lead one to consider the generation and evolution of the turbulence connected to the hyperbolicity of the flow equations.

In this paper we have considered only how the possible discontinuity in the initial conditions is propagated on the characteristic surfaces by means of the compatibility conditions [4].

2. General equations. Let us consider an unsteady perturbed plane flow of a viscous compressible fluid with a velocity vector $\mathbf{u}(\mathbf{x}, t)$ corresponding to a Mach number $M_{0}$ (evaluated with respect to a reference velocity $u_{0}$ ) small enough in order that the simplifications here afterwards indicated can be accepted; let us now define the motion, the equation deduced in [1] and applied in [1], [3]. This equation is

$$
\sigma^{*}\left[\frac{\partial}{\partial t}\left(\rho \frac{d u_{i}}{d t}+\frac{\partial P}{\partial x_{i}}\right)\right]=-\mu \rho \frac{d u_{i}}{d t}-\mu \frac{\partial P}{\partial x_{i}}+\mu^{2}\left(\frac{\partial^{2} u_{i}}{\partial x_{k} \partial x_{k}}+\frac{1}{3} \frac{\partial}{\partial x_{i}} \frac{\partial u_{l}}{\partial x_{l}}\right)
$$

where $\rho$ is the density, $P$ the pressure, and $\mu$ the viscosity coefficient; $\sigma^{*}=\sigma \rho$ is the quantity that in the constitutive equation gives the dependence of the stress tensor $\tau_{i k}$ on the variation in the time of the deformation rates tensor:

$$
\begin{aligned}
& \tau_{i k}=\mu\left(\gamma_{i k}-\frac{2}{3} \delta_{i k} \frac{\partial u_{l}}{\partial x_{l}}\right)-\sigma^{*}\left(\frac{\partial}{\partial t} \gamma_{i k}-\frac{2}{3} \delta_{i k} \frac{\partial^{2} u_{l}}{\partial t \partial x_{l}}\right)-P \delta_{i k} ; \\
& \gamma_{i k}=\frac{\partial u_{i}}{\partial x_{k}}+\frac{\partial u_{k}}{\partial x_{i}} ; \quad \delta_{i k}= \begin{cases}0 & \text { if } i \neq k \\
1 & \text { if } i=k .\end{cases}
\end{aligned}
$$

Let us denote

$$
\begin{aligned}
& P=\rho_{0} u_{0}^{2} \wp ; \quad u_{k}=u_{0} U_{k} ; \quad t=T \frac{L}{C_{\mathrm{so}}} ; \quad x_{k}=L X_{k} ; \quad \frac{\nu^{2}}{\sigma C_{\mathrm{so}}^{2}}=K^{*} ; \\
& \nu=\frac{\mu}{\rho} ; \quad \frac{4}{3} K^{*}+\frac{\wp}{\wp_{0}}=K ; \quad \sigma^{*}=\rho \sigma ;
\end{aligned}
$$


where the suffix zero applied to a quantity indicates the reference value of the same quantity; $C_{\text {so }}$ is the sound speed in a nonviscous fluid; $L$ is a characteristic length of the flow field; $\nu$ is the kinematic viscosity. The $\nu$ and $\sigma$ quantities are considered constant: taking into account their variation should complicate very much the formulae without altering the results at least qualitatively. The effect of the thermal conductivity is not considered and the evolution of the fluid is assumed to be isentropic. Under these conditions one deduces from Eq. (1)

$$
\begin{aligned}
\frac{\partial^{2} U_{i}}{\partial T^{2}} & +M_{0} U_{k} \frac{\partial^{2} U_{i}}{\partial X_{k} \partial T}+M_{0} \frac{\partial^{2} \wp}{\partial X_{i} \partial T}-K^{*}\left(\frac{\partial^{2} U_{i}}{\partial X_{k} \partial X_{k}}+\frac{1}{3} \frac{\partial^{2} U_{l}}{\partial X_{i} \partial X_{l}}\right) \\
& =-h\left(\frac{\partial U_{i}}{\partial T}+M_{0} U_{k} \frac{\partial U_{i}}{\partial X_{k}}+M_{0} \frac{\partial \wp}{\partial X_{i}}\right) ; \quad(i=1,2) .
\end{aligned}
$$

To Eq. (4) one has to adjoin the continuity equation that, under the same assumptions as those made for Eq. (4), can be written as

$$
\frac{\partial \wp}{\partial T}+\frac{\wp}{M_{0} \wp_{0}} \frac{\partial U_{k}}{\partial X_{k}}=0
$$

3. Characteristic surfaces. By expressing by means of Eq. (5) $\partial^{2} \wp / \partial X_{i} \partial T$ as a function of the derivatives of the $U_{k}$ one obtains

$$
L_{\mu}(\mathbf{U})=\sum_{1}^{2} \sum_{0}^{2}{ }_{i, k} A_{\mu \nu}^{i k} \frac{\partial^{2} U_{\nu}}{\partial X_{i} \partial X_{k}}+\phi_{\mu}(\mathbf{X}, \mathbf{U}, \wp)=0 \quad(i, k=0,1,2)
$$

where to compact the notations the quantity $T$ has been indicated with $X_{0}$, while the coefficients $A_{\mu \nu}^{i k}$ have the expressions given by Table (1).

\begin{tabular}{|c|c|c|c|c|c|c|}
\hline 00 & $\begin{array}{ll}0 & 1+ \\
1 & 0\end{array}$ & $\begin{array}{l}02+ \\
20\end{array}$ & 11 & $\begin{array}{l}12+ \\
21\end{array}$ & 22 & $(\mu \nu)$ \\
\hline 1 & $M_{0} U_{1}$ & $M_{0} U_{2}$ & $-K$ & 0 & $-K^{*}$ & 11 \\
\hline 0 & 0 & 0 & 0 & $-K_{1}$ & 0 & 12 \\
\hline 0 & 0 & 0 & 0 & $-K_{1}$ & 0 & 21 \\
\hline 1 & $M_{0} U_{1}$ & $M_{0} U_{2}$ & $-K^{*}$ & 0 & $-K$ & 22 \\
\hline
\end{tabular}

TABLE 1

$\left(A_{\mu \nu}^{i k}\right)$

$(i, k)$

$$
\begin{aligned}
& \left(K=\frac{\wp}{\wp_{0}}+\frac{4}{3} K^{*}\right) \\
& \left(K_{1}=\frac{\wp}{\wp_{0}}+\frac{1}{3} K^{*}\right)
\end{aligned}
$$


The functions $\phi_{\mu}$, at last, are given by the relations

$$
\left\{\begin{array}{l}
\phi_{1}=h\left[\frac{\partial U_{1}}{\partial X_{0}}+M_{0}\left(U_{1} \frac{\partial U_{1}}{\partial X_{1}}+U_{2} \frac{\partial U_{1}}{\partial X_{2}}+\frac{1}{\wp_{0}} \frac{\partial \wp}{\partial X_{1}}\left(1-\frac{1}{\wp_{0}} \frac{\partial \wp}{\partial X_{0}}\right)\right)\right] \\
\phi_{2}=h\left[\frac{\partial U_{2}}{\partial X_{0}}+M_{0}\left(U_{1} \frac{\partial U_{2}}{\partial X_{1}}+U_{2} \frac{\partial U_{2}}{\partial X_{2}}+\frac{1}{\wp_{0}} \frac{\partial \wp}{\partial X_{2}}\left(1-\frac{1}{\wp_{0}} \frac{\partial \wp}{\partial X_{0}}\right)\right)\right] .
\end{array}\right.
$$

The characteristic surfaces of Eq. (6) are defined by the characteristic equation

$$
Q\left(p_{0}, p_{1}, p_{2}\right)=\left|\sum_{0}^{2} i_{\mu} A_{\mu \nu}^{i k} p_{i} p_{k}\right|=0
$$

where

$$
p_{i}=\frac{\partial f}{\partial x_{i}}
$$

for any one characteristic surface $f(x)=$ const.

Therefore, on these surfaces, the following equation must be satisfied:

$$
\left|\begin{array}{c}
p_{0}^{2}+M_{0} p_{0}\left(U_{1} p_{1}+U_{2} p_{2}\right)-\left(K p_{1}^{2}+K^{*} p_{2}^{2}\right) ;-K_{1} p_{1} p_{2} \\
-K_{1} p_{1} p_{2} ; p_{0}^{2}+M_{0} p_{0}\left(U_{1} p_{1}+U_{2} p_{2}\right)-\left(K^{*} p_{1}^{2}+K p_{2}^{2}\right)
\end{array}\right|=0
$$

or

$$
\begin{aligned}
{\left[p_{0}^{2}+M_{0} p_{0}\left(U_{1} p_{1}+\right.\right.} & \left.\left.U_{2} p_{2}\right)-\left(K p_{1}^{2}+K^{*} p_{2}^{2}\right)\right] \\
& \cdot\left[p_{0}^{2}+M_{0} p_{0}\left(U_{1} p_{1}+U_{2} p_{2}\right)-\left(K^{*} p_{1}^{2}+K p_{2}^{2}\right)\right]-K_{1}^{2} p_{1}^{2} p_{2}^{2}=0
\end{aligned}
$$

Denoting

$$
Z=p_{0}^{2}+M_{0} p_{0}\left(U_{1} p_{1}+U_{2} p_{2}\right)
$$

Eq. $\left(10^{\prime}\right)$ becomes

$$
\left[Z-\left(K_{1} p_{1}^{2}+K^{*} p_{2}\right)\right]\left[Z-\left(K^{*} p_{1}^{2}+K p_{2}^{2}\right)\right]=K_{1}^{2} p_{1}^{2} p_{2}^{2}
$$

from which one obtains

$$
Z=Z_{i}=K^{(i)}\left(p_{1}^{2}+p_{2}^{2}\right) ; \quad\left(K^{(1)}=K\right) \quad\left(K^{(2)}=K^{*}\right)
$$

The quantities $Z_{i}$ are the roots of Eq. (12) that consequently may be written in the form $\left(Z-Z_{1}\right)\left(Z-Z_{2}\right)=0$, while Eq. $\left(10^{\prime}\right)$ can be split into the two equations

$$
p_{0}^{2}+M_{0} p_{0}\left(U_{1} p_{1}+U_{2} p_{2}\right)-Z_{i}=0 \quad(i=1,2) \text {. }
$$

One obtains

$$
p_{0}=-\frac{M_{0}\left(U_{1} p_{1}+U_{2} p_{2}\right)}{2} \pm \sqrt{\frac{M_{0}^{2}\left(U_{1} p_{1}+U_{2} p_{2}\right)^{2}}{4}+Z_{i}} .
$$

Since $Z_{i}>0$ the $p_{0}$ are real, so that the system (6) turns out to be totally hyperbolic, and to it corresponds a system of four families of characteristic surfaces, each one being 
defined by the partial differential equation of first order (15). By substitution for the $Z_{i}$ their expression (13) one deduces

$$
\frac{p_{0}^{2}}{p_{1}^{2}+p_{2}^{2}}+\frac{p_{0} M_{0}}{\sqrt{p_{1}^{2}+p_{2}^{2}}}\left(U_{1} \frac{p_{1}}{\sqrt{p_{1}^{2}+p_{2}^{2}}}+U_{2} \frac{p_{2}}{\sqrt{p_{1}^{2}+p_{2}^{2}}}\right)=K^{(i)}
$$

and since, if the normal to the wave surface is oriented in the sense of increasing $f$, $-p_{0} / \sqrt{p_{1}^{2}+p_{2}^{2}}$ defines the displacement velocity $C_{s}$ of the wave, while

$$
M_{0}\left(\frac{U_{1} p_{1}}{\sqrt{p_{1}^{2}+p_{2}^{2}}}+\frac{U_{2} p_{2}}{\sqrt{p_{1}^{2}+p_{2}^{2}}}\right)
$$

gives the component $U_{n} M_{0}$ of the fluid velocity normal to the wave surface, it is $C_{s}=$ $-U_{n} M_{0}=C_{p}$, in which $C_{p}$ defines the propagation velocity of the wave in the flow stream, so that it appears that

$$
C_{s} C_{p}=K^{(i)}
$$

Equation (17) defines the variation law of the propagation velocity of each element of the wave surface with the orientation of said element. One has to observe that for $M_{0}=0$ and $i=1$, Eq. (17) is coincident with the result obtained in [1], while for $M_{0}=0$ and Prandtl number $P_{r}=1$, Eq. (17), for $i=1,2$, coincides with that obtained in [3].

4. Dynamic compatibility conditions. Discontinuity parameter. It is known that solutions of the system (4) may exist, for which the derivatives of the first order of velocity are continuous but the derivatives of second order are discontinuous.

These have to satisfy the geometric-kinematic conditions [4] given by the relations

$$
\Delta \frac{\partial^{2} U_{i}}{\partial X_{i} \partial X_{k}}=h_{i} p_{i} p_{k} ; \quad(i, k=0,1,2)
$$

and, therefore, by the dynamic compatibility conditions, one obtains

$$
\left\{\begin{array}{l}
h_{1} p_{0}^{2}+M_{0} h_{1} p_{0}\left(U_{1} p_{1}+U_{2} p_{2}\right)-K h_{1} p_{1}^{2}-K^{*} h_{1} p_{2}^{2}-K_{1} h_{2} p_{1} p_{2}=0 \\
K_{1} h_{1} p_{1} p_{2}+h_{2} p_{0}^{2}+M_{0} h_{2} p_{0}\left(U_{1} p_{1}+U_{2} p_{2}\right)-K^{*} h_{2} p_{1}^{2}-K h_{2} p_{2}^{2}=0
\end{array}\right.
$$

and the system has proper solutions as a consequence of Eq. (10).

Denote by $\mathbf{h}$ the vector parameter of the discontinuity defined by

$$
\mathbf{h}=h_{1} \mathbf{i}_{1}+h_{2} \mathbf{i}_{2}
$$

$\mathbf{i}_{1}$ and $\mathbf{i}_{2}$ being the unit vectors along the directions of the axes $X_{1}$ and $X_{2}$ and write

$$
g^{2}=p_{1}^{2}+p_{2}^{2} ; \quad \alpha_{k}=\frac{p_{k}}{g} \quad(k=1,2) .
$$

This is

$$
\sum_{1}^{2} h_{k} p_{k}=g \sum h_{k} \alpha_{k}=g h_{n}
$$


$h_{n}$ being the component of the vector $\mathbf{h}$ in the point $\left(X_{1}, X_{2}\right)$ and having therefore components along the axes equal to $\alpha_{k}$. One deduces

$$
\begin{aligned}
& -K h_{1} p_{1}^{2}-K^{*} h_{1} p_{2}^{2}-K_{1} h_{2} p_{1} p_{2}=-K g^{2} \alpha_{1} h_{n}+K^{*} g^{2} \alpha_{2} h_{\tau} \\
& -K h_{2} p_{2}^{2}-K^{*} h_{2} p_{1}^{2}-K_{1} h_{1} p_{1} p_{2}=-K g^{2} \alpha_{2} h_{n}-K^{*} g^{2} \alpha_{1} h_{\tau}
\end{aligned}
$$

where $h_{\tau}$ is the component of the vector $\mathbf{h}$ in the plane $\left(X_{1}, X_{2}\right)$ normal to the vector $\mathbf{n}$, and therefore

$$
\mathbf{h}\left(p_{0}^{2}+M_{0} p_{0} U_{n}\right)=K g^{2} h_{n} \mathbf{n}+K^{*} g^{2} h_{\tau} \tau .
$$

The discontinuity vector $\mathbf{h}$ has therefore both a longitudinal and a transversal component.

5. Bicharacteristics. Characteristic cone. Normal cone. From the first-order partial differential equation (16) defining every single family of characteristic surface, one obtains easily the first-order ordinary equations that determine the corresponding characteristic lines, i.e., the bicharacteristics of the system (6). Writing Eq. (16) in the form

$$
\psi\left(X_{i}, p_{i}\right)=\sum_{0}^{2} \alpha_{i k} p_{i} p_{k}=0
$$

where the dependence of the function $\psi$ on the coordinates $X_{i}$ is due only to the dependence of $\psi$ on $U_{i}$, one is able to define the bicharacteristics by the equations

$$
\frac{d X_{i}}{d s}=\psi_{i} ; \quad \frac{d p_{i}}{d s}=-\psi_{U_{j}} \frac{\partial U_{j}}{\partial x_{i}} \quad(i=0,1,2) \quad\left(\psi_{U_{j}}=\frac{\partial \psi}{\partial U_{i}} ; \quad \psi_{i}=\frac{\partial \psi}{\partial p_{i}}\right)
$$

and, therefore,

$$
\begin{array}{clrl}
\frac{d X_{0}}{d s}=2 p_{0}+M_{0}\left(U_{1} p_{1}+U_{2} p_{2}\right), & \frac{d X_{1}}{d s}=M_{0} p_{0} U_{1}-2 p_{1} K^{(i)}, \\
\frac{d X_{2}}{d s}=M_{0} p_{0} U_{2}-2 p_{2} K^{(i)}, & \frac{d p_{i}}{d s}=-M_{0} p_{0} p_{j} \frac{\partial U_{j}}{\partial X_{i}}
\end{array}
$$

$s$ being a parameter proportional to the length of the arc between two points of the bicharacteristics at issue. Now, the quantities $p_{i}$ at a general point $P$ of the characteristic surface $f\left(X_{i}\right)=$ const. are the direction coefficients of the straight-line normal to this surface at the point $P$, and if one considers the vectors whose components are $p_{i}$ and have therefore the direction of these normals in all the points of the surface $f\left(X_{i}\right)=$ const. emanating from the origin of rectangular $p_{i}$ space, their end points lie on the surface whose equation is Eq. (16) and that defines a quadratic cone: this cone, since its generating rays are parallel to the normal of the characteristic surface in question, is the so-called "normal cone": actually, it is

$$
p_{i} \frac{d X_{i}}{d s}=p_{i} \psi_{i}
$$


and since the function $\psi$ is a homogeneous function of first degree with respect to the $p_{i}$, it is $p_{i} \psi_{i}=\psi=0$. By Eq. (25) then, one deduces

$$
\frac{d X_{i}}{d s}=a_{i k} p_{k}
$$

and, if $a_{i k}^{\prime}$ is the inverse matrix of the matrix $a_{i k}$, it becomes

$$
p_{k}=a_{k i}^{\prime} \frac{d X_{i}}{d s}
$$

substituting (29) into (25) one obtains

$$
a_{i k}^{\prime} \frac{d X_{i}}{d s} \frac{d X_{k}}{d s}=0=a_{i k}^{*} \frac{d X_{i}}{d s} \frac{d X_{k}}{d s}
$$

which defines a quadratic cone: this is the envelope of the planes tangent to all the characteristic surface elements passing through $P$ and constitutes the "Monge cone" while the $a_{i k}^{\prime}$ is the adjoint matrix of the matrix $a_{i k}$.

6. Variation law of the velocity on the characteristic surfaces. Let us assume a coordinate system $\left(\xi_{0}, \xi_{1}, \xi_{2}\right)$ defined as follows:

$$
\xi_{0}=f\left(X_{0}, X_{1}, X_{2}\right) ; \quad \xi_{1}=X_{1} ; \quad \xi_{2}=X_{2}
$$

so that $\xi_{0}=$ const. represents a family of characteristic surfaces. With the new coordinate system, Eqs. (6) become

$$
L_{u}(\mathbf{U})=\sum_{\nu=1}^{2} \sum_{0}^{2}{ }_{i, k} \alpha_{\mu \nu}^{i k} \frac{\delta U_{\nu, \xi_{k}}}{\delta \xi_{i}}=\chi_{\mu}(\xi, \mathbf{U}) \quad\left(U_{\nu, \xi_{k}}=\frac{\partial U_{\nu}}{\partial \xi_{k}}\right)
$$

where the coefficients $\alpha_{\mu \nu}^{i k}$ are given by

$$
\alpha_{\mu \nu}^{i k}=\sum_{0}^{2}{ }_{j, l} A_{\mu \nu}^{j l} \tau_{i j} \tau_{k l}
$$

where

$$
\tau_{i j}=\frac{\partial \xi_{i}}{\partial X_{j}}
$$

and where the notation $\delta$ is used to point out that the derivatives are inner derivatives, that is, calculated on the surface itself. The expression of the coefficients $\alpha_{\mu \nu}^{i k}$ is given by Table (2). 


\begin{tabular}{|c|c|c|c|c|c|c|}
\hline 00 & $\begin{array}{ll}0 & 1 \\
1 & 0\end{array}$ & $\begin{array}{ll}0 & 2 \\
2 & 0\end{array}$ & 11 & $\begin{array}{ll}1 & 2 \\
2 & 1 \\
\end{array}$ & 22 & $\mu \nu$ \\
\hline $\begin{array}{c}-K p_{1}^{2}-K^{*} p_{2}^{2}+p_{0}^{2} \\
+M_{0} p_{0}\left(U_{1} p_{1}+U_{2} p_{2}\right)\end{array}$ & $-K p_{1}+\frac{1}{2} M_{0} p_{0} U_{1}$ & $-K^{*} p_{2}+\frac{1}{2} M_{0} p_{0} U_{2}$ & $-K$ & 0 & $-K^{*}$ & $\begin{array}{lll}1 & 1\end{array}$ \\
\hline$-K_{1} p_{1} p_{2}$ & $-\frac{1}{2} K_{1} p_{2}$ & $-\frac{1}{2} K_{1} p_{1}$ & 0 & $\frac{1}{2} K_{1}$ & 0 & 12 \\
\hline$-K_{1} p_{1} p_{2}$ & $-\frac{1}{2} K_{1} p_{2}$ & $-\frac{1}{2} K_{1} p_{1}$ & 0 & $\frac{1}{2} K_{1}$ & 0 & 21 \\
\hline $\begin{array}{c}-K^{*} p_{1}^{2}-K p_{2}^{2}+p_{0}^{2} \\
+M_{0} p_{0}\left(U_{1} p_{1}+U_{2} p_{2}\right)\end{array}$ & $-K^{*} p_{1}+\frac{1}{2} M_{0} p_{0} U_{1}$ & $-K p_{2}+\frac{1}{2} M_{0} p_{0} U_{2}$ & $-K^{*}$ & 0 & $-K$ & 22 \\
\hline
\end{tabular}

Writing $f(\mathbf{X})=\varphi(\xi)$, therefore $\varphi(\xi)=$ const. is the equation of any characteristic surface in the new coordinates $\xi_{i}$. It must be, because of Eq. (8),

$$
\left|\sum_{0}^{2} \alpha_{\mu \nu}^{i k} \varphi_{i} \varphi_{k}\right|=0
$$

The equations that define the variation law of the velocity on the characteristic surface can be deduced by Eq. (32), observing that

$$
\sum_{1}^{2}{ }_{\nu} \alpha_{\mu \nu}^{00} \frac{\partial^{2} U_{\nu}}{\partial \xi_{0}^{2}}=0
$$

At the left side of Eq. (32) only the derivatives $U_{\nu, \xi_{i}}$ with respect to $\xi_{1}$ and $\xi_{2}$ remain, and these can be calculated along the bicharacteristics of the surface in question.

Since

$$
\frac{d \xi_{i}}{d \sigma}=\sum_{0}^{2} k \frac{d X_{i}}{d s} \tau_{i k}=\frac{d X_{i}}{d s} \quad(i=1,2)
$$

the corresponding direction coefficients are given by the same relations (27). For each point $P$ of the field, considering the four characteristic surfaces passing through this point, one can write eight equations like Eq. (32), and these, in addition to the continuity equation (5), that is already in the characteristic form, constitute a system of nine equations in the nine unknown functions

$$
\frac{\partial U_{\kappa}}{\partial X_{i}}, \quad \frac{\partial \wp}{\partial X_{i}} \quad(i=0,1,2) \quad(\kappa=1,2) .
$$

These equations can be solved numerically "step-by-step" using a method similar to that used for the Cauchy problems that have been considered in (1) and (3).

7. Conclusions. Even for the problem considered in this paper the system of equations representing the flow is totally hyperbolic, so that any perturbation that is produced 
in any point propagates by means of waves corresponding to the families of characteristic surfaces passing through that point. The numerical solution of the Cauchy problem can be obtained, at least conceptually, by means of a "step-by-step" method as in the cases considered in [1] and [3]; however, it requires much more complicated calculations because of the greater complexity and greater number of equations. Besides, the multiplicity of the propagation waves and their different displacement speeds lead to an interference between the same waves, and generation of discontinuity, that produces easily a chaotic motion. The difficulties now mentioned increase very much in the case of three-dimensional flow, in which one should have five systems of characteristic surfaces and sixteen equations to determine the sixteen unknown functions

$$
\frac{\partial U_{\nu}}{\partial X_{i}}, \quad \frac{\partial p}{\partial X_{i}} \quad(i=0,1,2,3) \quad(\nu=1,2,3) .
$$

\section{REFERENCES}

[1] C. Ferrari, On the propagation of small perturbations in viscous compressible fluid, Acta Mech. [Suppl.] 3, 1-16 (1992)

[2] C. Cattaneo, Sulla conduzione del calore, Seminario matematico e fisico, Università di Modena, Società Tipografica Modenese, 1948

[3] C. Ferrari, The effect of the thermal conductivity on the propagation of small perturbations in viscous compressible fluid, Acta Mech. [Suppl.] 4, 305-311 (1994)

[4] T. Levi-Civita, Caracteristiques des Systèmes Differentiels et Propagation des Ondes, Paris Librairies Felix Akan, 1932, pp. 56-61

This article is the last paper of Prof. Carlo Ferrari and was published after his death which occurred at the age of 93, on May 21st, 1996.

Scholars and friends, with great affection, remember the illustrious scientist and his great contribution to the entire range of Fluid Mechanics. 